\title{
Zirkulierende Tumorzellen in der Diagnose und Therapie des Mammakarzinoms
}

\author{
Gesprächsleitung: Tanja Fehm ${ }^{a}$ \\ Teilnehmer: Andreas Hartkopf ${ }^{b} \quad$ Wolfgang Jannic \\ Erich Solomayer $^{d}$ Brigitte Rack ${ }^{\mathrm{e}}$ Volkmar Müller ${ }^{f}$ \\ aUniversitätsfrauenklinik Düsseldorf, \\ 'Universitätsfrauenklinik Tübingen, \\ 'Universitätsfrauenklinik, Ulm, \\ ${ }^{d}$ Klinik für Frauenheilkunde, Geburtshilfe und Reproduktionsmedizin, Homburg/Saar, \\ eKlinik und Poliklinik für Frauenheilkunde und Geburtshilfe, Klinikum Innenstadt, München, \\ fUniversitätsfrauenklinik Hamburg-Eppendorf, Germany
}

\section{Frage 1: Was ist heute für Sie die beste Methode zum CTC-Nachweis im Blut?}

Hartkopf: Das CellSearch ${ }^{\circledR}$-System (Veridex, Janssen Diagnostics, LLC, Raritan, NJ, USA), da die prognostische Relevanz der Detektion von zirkulierenden Tumorzellen (CTC) mittels CellSearch, insbesondere in der metastasierten Situation, in einer Vielzahl unabhängiger Studien nachgewiesen werden konnte.

Janni: Derzeit ist die einzige zugelassene Methode der halbautomatisierte immunzytochemische Nachweis mit der CellSearch-Methode, die auf den verschiedenen Evidenzniveaus validiert wurde. Für diese Methode besteht ein Level of Evidence I. Andere, weniger validierte Methoden sind sicher wissenschaftlich sehr interessant, aber derzeit noch nicht für den kliniknahen Bereich geeignet.

Solomayer: Es gibt nur eine zugelassene Methode und zwar die CellSearch-Methode. Sie kann zuverlässig die Tumorzellen epithelialer Herkunft durch Antikörper gegen CD45-, EpCAM+, Zytokeratine 8, 18 und/oder Zytokeratin 19 im Blut detektieren. Die amerikanische Kontrollbehörde FDA hatte die Methode nicht nur bei Brustkrebs sondern auch bei metastasiertem Darm- und Prostatakrebs zugelassen.

Rack: Für die klinische Anwendung ist CellSearch sicher am besten in zahlreichen Studien mit teilweise großen Fallzahlen und multizentrisch validiert. Für Forschungsansätze mögen andere Methoden je nach Fragestellung Vorteile bieten.

Müller: Eine entscheidende Anforderung an ein in der klinischen Praxis anwendbares System zum Nachweis von
CTC ist die Reproduzierbarkeit von Ergebnissen in einem klinischen Multicenter-Setting, das auch den Versand von Proben und die Bestimmung in mehreren Zentren beinhaltet. Dieses konnte meines Erachtens am besten mit dem System CellSearch zur standardisierten und weitgehend automatisierten Anreicherung mit immunzytochemischem Nachweis von CTC erzielt werden.

\section{Frage 2: Welche Probleme gibt es derzeit beim Nachweis von CTC im Blut?}

Hartkopf: Ein Problem ist die wahrscheinlich geringe Sensitivität. Bei der Detektion von CTC mittels CellSearch werden Cytokeratin-positive Zellen angereichert. Es kann allerdings davon ausgegangen werden, dass Tumorzellen im Blut zirkulieren, die kein Cytokeratin exprimieren.

Janni: Die Herausforderung für die Zukunft werden meines Erachtens hauptsächlich die Erhöhung der Sensitivität für den Nachweis von geringer zirkulierender Tumorlast bei Primärerkrankung und die Charakterisierung von Einzelzellen sein. Insbesondere bei der Charakterisierung folgt der methodische Fortschritt nur langsam den klinischen Ansprüchen.

Solomayer: Die Detektion beim metastasierten Mammakarzinom ist sehr gut. In der adjuvanten Situation müsste die Methode sensitiver die Tumorzellen nachweisen. Ebenfalls wäre es wünschenswert, mehr Eigenschaften der detektierten Tumorzellen nachweisen zu können. Z.B. wird derzeit das metastatische Potential der nachgewiesenen Tumorzellen nicht erfasst.

\section{KARGER \\ Fax +497614520714 \\ Information@Karger.com}

www.karger.com (c) 2013 S. Karger GmbH, Freiburg

$1661-3791 / 13 / 0085-0375 \$ 38.00 / 0$

Accessible online at:

www.karger.com/brc
Prof. Dr. Tanja Fehm

Universitätsfrauenklinik Düsseldorf

Moorenstr. 5

40225 Düsseldorf, Germany

Tanja.Fehm@med.uni-duesseldorf.de 
Rack: Das größte Problem ist die relativ geringe Sensitivität der Methode, bisher ist die Multimarker-Charakterisierung nicht etabliert.

Müller: Ein Problem ist die möglicherweise nicht ausreichende Sensitivität beim Nachweis der Zellen. Die Detektion erfolgt meist basierend auf der Expression bestimmter Antigene und hier gelingt es sicher mit keinem Verfahren, alle Zellen mit ausreichender Spezifität nachzuweisen.

\section{Frage 3: Wie sehen Sie den aktuellen Stellenwert der CTC in der adjuvanten Situation?}

Hartkopf: Erste große Studien konnten auch hier eine prognostische Relevanz zeigen. Allerdings ist die Positivitätsrate sehr niedrig. Dies ist insbesondere für weiterführende Studien (z.B. Charakterisierung von CTC) problematisch, da eine hohe Zahl von Patientinnen analysiert werden muss, um valide Ergebnisse zu erzielen. Das unter Punkt 2 genannte Problem der geringen Sensitivität ist daher insbesondere in der adjuvanten Situation von Bedeutung.

Janni: Die Evidenzbasis nimmt in diesem Setting zu, und wird zunehmend für die Zellcharakterisierung interessant werden. Adjuvante Therapiekonzepte werden, abseits von Hormonrezeptor- und HER2-Status, immer noch wenig individualisiert indiziert. Hier gibt es einen großen Bedarf.

Solomayer: Aktuell sind einige interessante Daten in der adjuvanten Situation vorhanden und weitere Studien zu der klinischen Bedeutung laufen derzeit. Auch wenn aktuell die Wertigkeit der Methode in der adjuvanten Situation noch nicht etabliert ist, sind die vorhandenen Daten vielversprechend.

Rack: Daten zur prognostischen Relevanz liegen vor. Allerdings ist bisher eine tatsächliche Verbesserung des Krankheitsverlaufes durch den Einsatz von CTCs nicht erwiesen. Daher sollte das Verfahren m.E. in der Adjuvanz ausschließlich in klinischen Studien eingesetzt werden.

Müller: Einzelne Studien konnten eine prognostische Relevanz des Nachweises von CTC bei Patientinnen mit primärem Mammakarzinom zeigen, allerdings gibt es keine Bestätigung, dass aus diesen vorläufigen Ergebnissen therapeutische Konsequenzen zum Vorteil für die Patientinnen gezogen werden können. Diese Fragestellung wird unter anderem gegenwärtig in der SUCCESS-C Studie und in der 2013 startenden Studie «TREAT CTC» untersucht.

\section{Frage 4: Welche Bedeutung haben CTC aktuell beim metastasierten Mammakarzinom?}

Hartkopf: Die Bedeutung ist dreifaltig: Erstens können Patientinnen selektiert werden, die ein erhöhtes Risiko für ein Fortschreiten der Erkrankung aufweisen und gegebenenfalls eine aggressivere Therapie benötigen. Zweitens scheinen CTC das Ansprechen auf eine Therapie schneller und gegebenenfalls besser aufzuzeigen als die üblicherweise durchgeführten radiologischen Kontrollen und drittens ist die Phänotypisierung von CTCs ein vielversprechendes Instrument das Ansprechen auf moderne Therapien (z.B. die HER2-zielgerichtete Therapie) vorherzusagen.

Janni: Durch die Phänotypisierung der CTC werden neue Therapieansätze ermöglicht, deren klinischer Nutzen noch durch prospektive Studien nachgewiesen werden muss. Als früher Ansprechmarker sind CTC schon seit vielen Jahren evidenzbasiert.

Solomayer: Durch die Phänotypisierung der CTC werden neue Therapieansätze ermöglicht, deren klinischer Benefit allerdings noch durch prospektive Studien nachgewiesen werden muss.

Rack: Die CTC können über die Bestimmung der CTC-Anzahl eine frühzeitige Abschätzung der Therapieeffektivität ermöglichen. Außerdem ist eine wiederholte Untersuchung des Tumorphänotyps möglich. Aktuelle randomisierte Studien haben die klinische Relevanz einer Therapieumstellung basierend auf CTC zum Gegenstand, allerdings liegen noch keine publizierten Daten vor. Jedoch erscheint hier auf Basis der bereits publizierten nicht-randomisierten Daten ein Einsatz in klinischen Einzelfällen gerechtfertigt, wenn keine vergleichbare Diagnostik zur Verfügung steht.

Müller: Die prognostische Relevanz in der metastasierten Situation konnte durch eine Vielzahl von Studien bestätigt werden und gilt als gesichert unter Verwendung von CellSearch und des Cut-off-Levels von 5 CTC in 7,5 ml Blut. Das frühe Erkennen einer Progression unter Therapie ist eine weitere relevante Fragestellung. Hier konnten Christofanilli et al. zeigen, dass die Tumorzellpersistenz unter laufender Erstlinientherapie mit einer besonders schlechten Prognose assoziiert ist. Derzeit soll in der amerikanischen Studie SWOG S0500 überprüft werden, ob ein frühzeitiger Therapiewechsel bei Tumorzellpersistenz im Vergleich zum Therapiewechsel erst bei bildgebend sichtbarem Progress mit einem Überlebensvorteil assoziiert ist. Eine Testung mit einem standardisierten Verfahren kann meines Erachtens schon jetzt in bestimmten klinischen Situationen sinnvoll sein. Solche Therapiesituationen sind beispielsweise schlechte Verträglichkeit 
einer laufenden Therapie mit dem Ziel der Vermeidung einer unnötigen Toxizität oder eine Risikoabschätzung vor dem Hintergrund einer Entscheidung über die Aggressivität einer Therapie in der metastasierten Situation.

\section{Frage 5: Welches ist derzeit aus Ihrer Sicht die wichtigste CTC-Studie, um die Bedeutung der CTC weiter zu spezifizieren?}

Hartkopf: Die multizentrische DETECT-III-Studie (www.detetct-studie.de). Hier werden CTCs in der metastasierten Situation charakterisiert und es wird evaluiert, ob HER2-negative Brustkrebspatientinnen mit HER2-positiven CTC von einer Therapie mit Lapatinb profitieren (vgl. auch Frage 3).

Janni: Die DETECT-Studien, da sie das Potential der Prädiktion auf dem Boden der Phänotypisierung von CTC ausschöpfen und Patientinnen eine individuellere Therapie erlauben

\section{Solomayer: Die DETECT-Studien.}

Rack: Aus meiner Sicht sind dies die SWOG-Studie, die DETECT-Studien und die TREAT-CTC-Studie (Benefit von Trastuzumab bei CTC-positiven Patientinnen in der Adjuvanz).

Müller: Eine methodische Studie der DETECT-Studiengruppe der AG Tumorzelldissemination der Deutschen Gesellschaft für Senologie zur Expression von HER2 auf CTC ist abgeschlossen, eine klinische Studie zum Einsatz einer Therapie mit Lapatinib bei Patientinnen mit HER2-negativem Primärtumor und HER2-positiven CTC läuft gegenwärtig (DETECT III). Darüber hinaus sind die Ergebnisse der oben erwähnten Studie SWOG S0500 von klinischer Relevanz.

\section{Frage 6: Wo wird die Zukunft der CTC aus Ihrer Sicht langfristig liegen?}

Hartkopf: Da CTCs als ursächlich für die Metastasenbildung angesehen werden, liegt das größte Potential meiner Ansicht nach in der molekularen Charakterisierung von CTCs. Das Verständnis der molekularen Grundlagen einer Krankheitsprogression ist unbedingte Voraussetzung für die Entwicklung neuer und effektiverer Therapeutika.

Janni: Die zukünftige Rolle der CTC liegt aus meiner Sicht in der therapierelevanten Phänotypisierung der aktuellen Erkrankungssituation zur Individualisierung der Therapie.
Solomayer: In der metastasierten Situation hat der CTC-Nachweis eine klinische Bedeutung. Durch den Einsatz der CTCs kann ein Ansprechen oder Nichtansprechen auf eine Chemotherapie frühzeitig erkannt werden. In einigen Studien werden biologische Eigenschaften der Tumorzellen untersucht, um neue Therapien zu entwickeln. Ein Beispiel dafür ist die DETECT-Studie, in der die HER-2-Expression auf den CTC in ein neues Studiendesign integriert wird.

Rack: Zukünftig wird die individualisierte Therapieführung sowohl in der Postadjuvanz als auch in der metastasierten Situation, basierend auf der Phänotypisierung von CTC, eine wichtige Rolle spielen.

Müller: Da die zirkulierenden Tumorzellen den Phänotyp der Zellen mit dem Potential der hämatogenen Metastasierung widerspiegeln, kann möglicherweise durch die Charakterisierung von CTC eine Therapieoptimierung erfolgen. Dies ist neben den oben geschilderten Anwendungen eine Perspektive klinischer Anwendung.

\section{Teilnehmer:}

Dr. Andreas Hartkopf

Universitätsfrauenklinik Tübingen

Calwer Str. 7

72076 Tübingen, Germany

Andreas.hartkopf@med.uni-tuebingen.de

Prof. Dr. Wolfgang Janni

Universitätsfrauenklinik, Ulm

Prittwitzstr. 43

89075 Ulm, Germany

wolfgang.janni@uniklinik-ulm.de

Prof. Dr. Erich Solomayer

Klinik für Frauenheilkunde, Geburtshilfe und Reproduktionsmedizin Kirrbergerstr. 100

66421 Homburg/Saar, Germany

erich.solomayer@uniklinikum-saarland.de

PD Dr. Brigitte Rack

Klinik und Poliklinik für Frauenheilkunde und Geburtshilfe

Klinikum Innenstadt

Maistrasse 11

80337 München, Germany

brigitte.rack@med.uni-muenchen.de

Prof. Dr. Volkmar Müller

Universitätsfrauenklinik Hamburg-Eppendorf

Martinistraße 52

20246 Hamburg, Germany

vmueller@uke.de 